نحوه توارث مقاومت به بيمارى زنَّ قهوهاى در كَندم نان

با روش تجزيه ميانكين نسلها

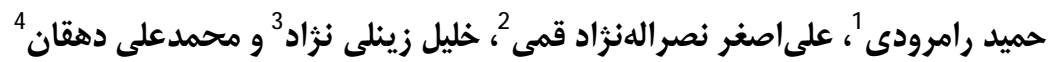

2 1 - 2 دانشجوى كارشناسى ارشد و استاديار، اصلاح نباتات دانشكاه علوم كشاورزى و منابع طبيعى گركان

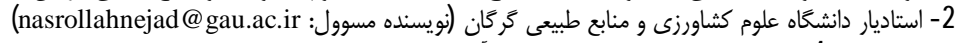

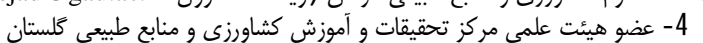

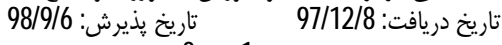

صفحه: 1 تا 8 تار

جكيده

زنحَ قهوهاى كندم توسط قارج

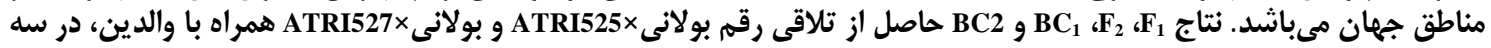

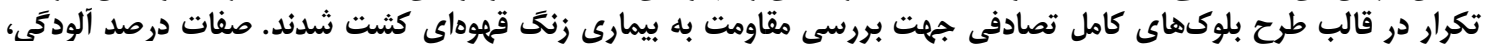

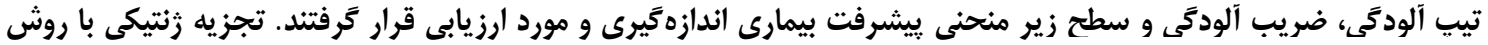

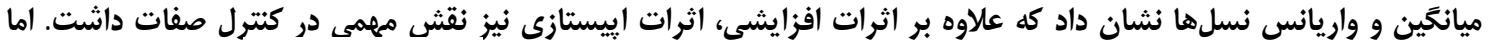

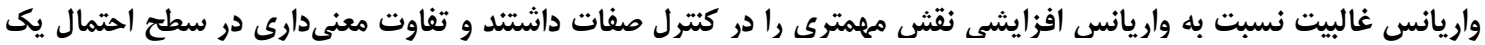

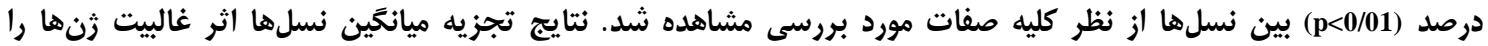

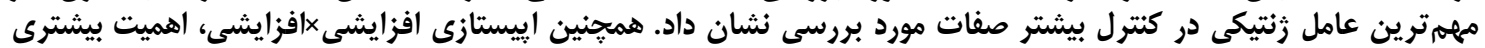

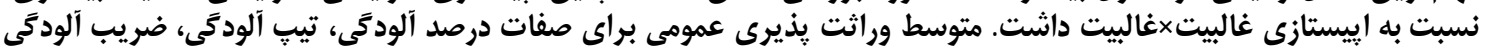

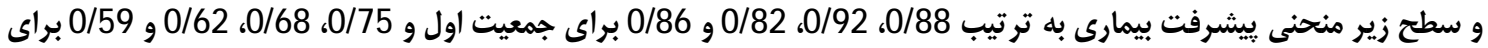

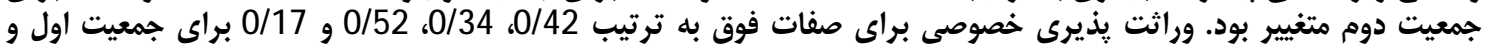

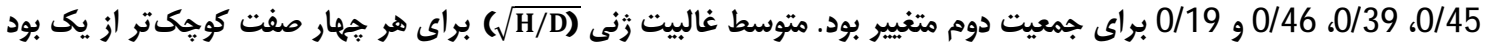

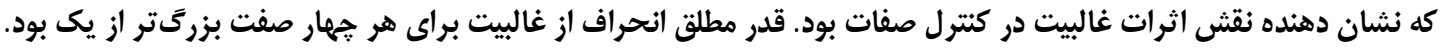

وازههاى كليدى: إييستازى، واريانس غالبيت، وراثتيذيرى خصوصى، وراثت يذيرى عمومى

مبارزه با بيمارى زنخ قهوهاى محسوب مى رَّدد. مطالعات

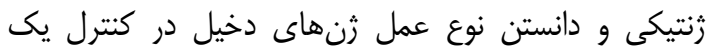

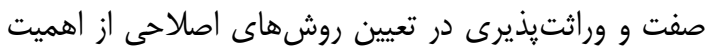

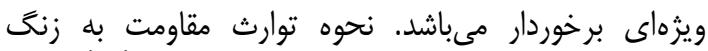

قهوهاى در ارقام مختلفى از گتندم تعيين شده است نوره (24).

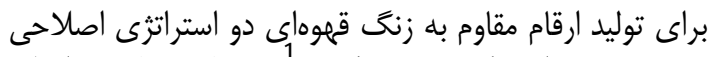

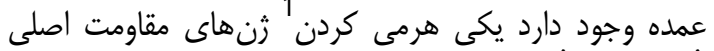

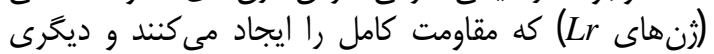

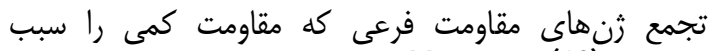

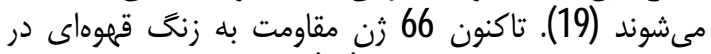

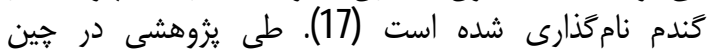

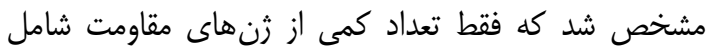
ئابل

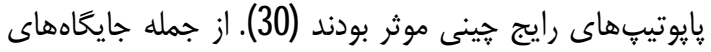

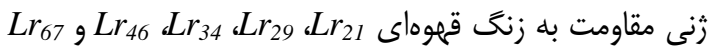

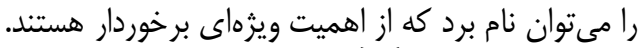

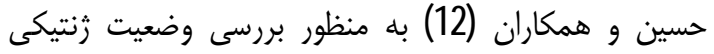

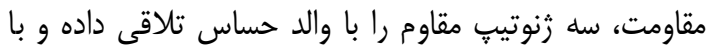

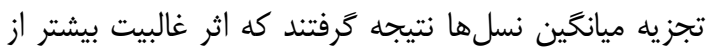

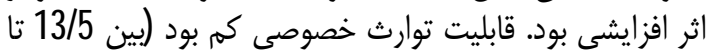

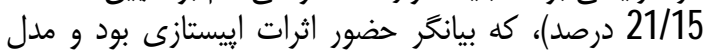

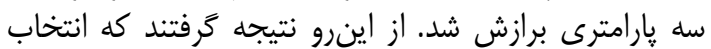

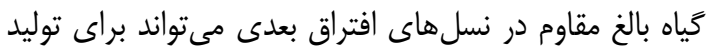

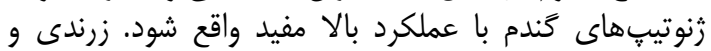

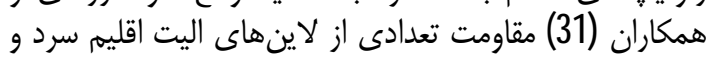

مقدمه عامل بيمارى زنخ قهوهاى گَندم قارجى است بإ نام علمى Puccinia recondita f. sp. tritici كه به به بيمارى

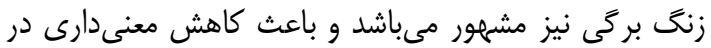

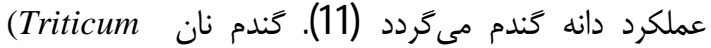
aestivum L.)

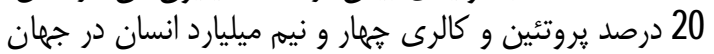

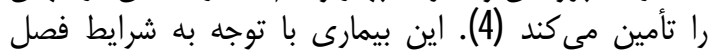

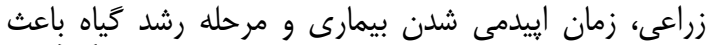

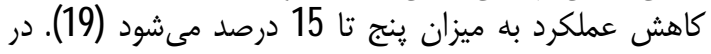

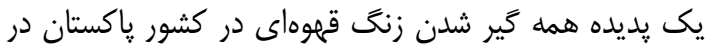

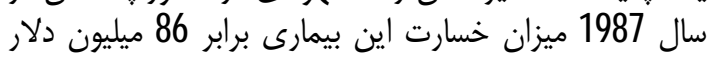

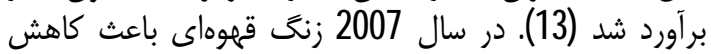

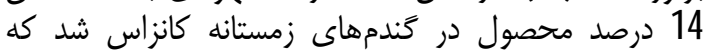

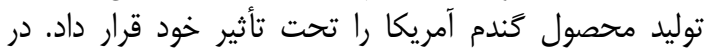

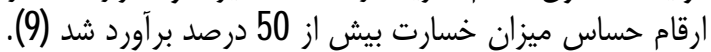

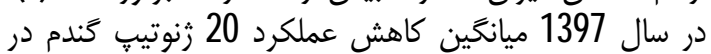

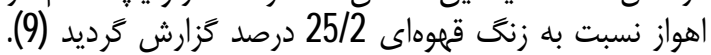

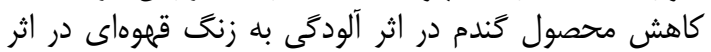

كاهش دانه در سنبله و وزن هول هزار دار دانه است (22).

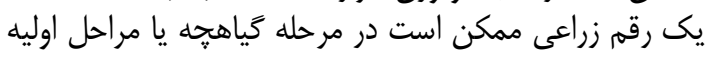

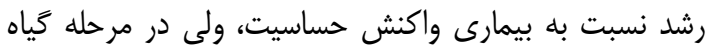

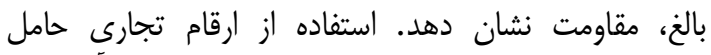

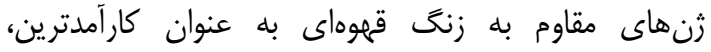

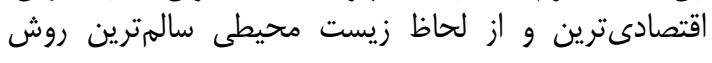


يخش كننده بيمارى كشت گرديد. به دليل مساعد بودن منطقه

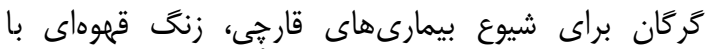

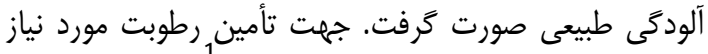

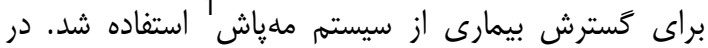

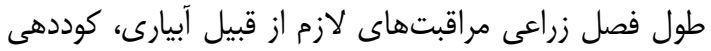

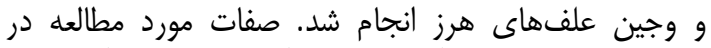

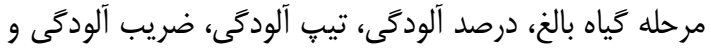

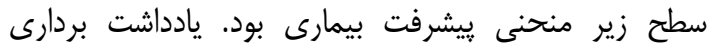

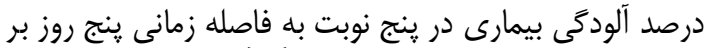

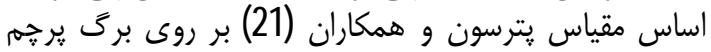

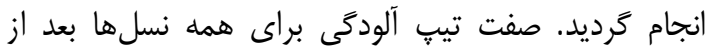

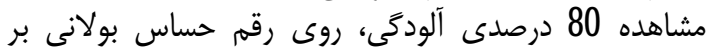

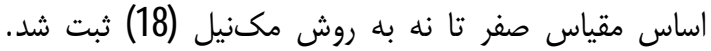

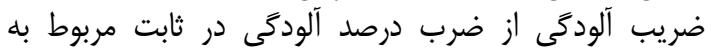

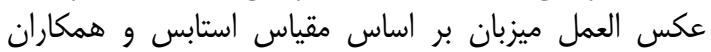
(Immune=0， R=0.2， MR=0.4, M=0.6, (26) بS=0.8, S=1) يبشرفت بيمارى با استفاده از فرمول1 ويل ويلكاكسون و همكاران

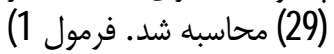
AUDPC $=\sum_{\mathrm{i}=1}^{\mathrm{k}} 1 / 2(\mathrm{Si}+\mathrm{Si}+1) * \mathrm{~d}$

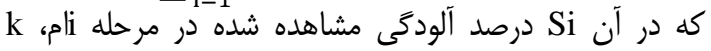

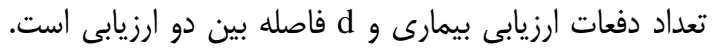

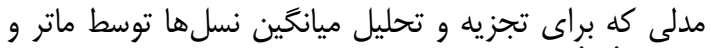

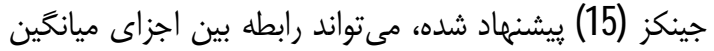

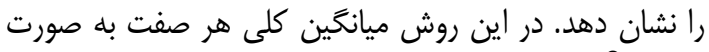
فرمول 2 نشان داده مى إنود.

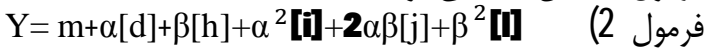
اجزاء فرمول عبارتند از:

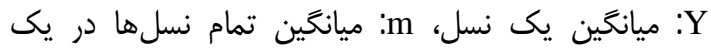

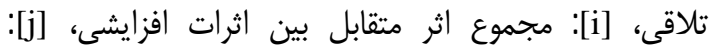

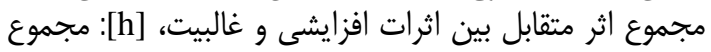

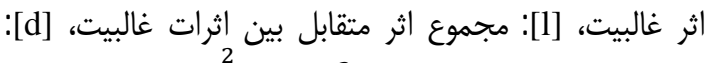

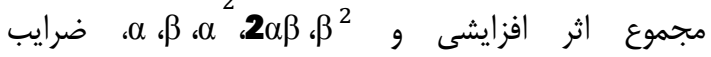

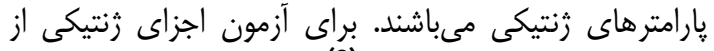

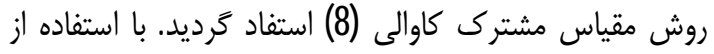

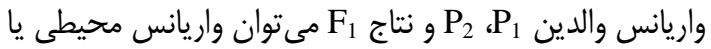

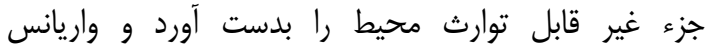
نسل هاى Fe $\sigma_{F_{1}}^{2}=\mathrm{E}_{1} \sigma_{p_{2}}^{2}=\mathrm{E}_{1} \sigma_{p_{1}}^{2}=\mathrm{E}_{1} \quad$ فرمول) $\sigma_{F_{2}}^{2}=\frac{1}{2} \mathrm{~A}+\frac{1}{4} \mathrm{D}+\mathrm{E}_{1}$ فرمول5) (6رول) فرمول6)

با استفاده از اميد رياضى اجزاى واريانس نسلها مىتوان

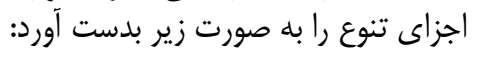

$$
\begin{aligned}
& \mathrm{E}=\frac{1}{4}\left(\sigma_{P_{1}}^{2}+\sigma_{P_{2}}^{2}+2 \sigma_{F_{1}}^{2}\right) \\
& \text { فرمول7) } \\
& \mathrm{A}=4 \sigma_{F_{2}}^{2}-2\left(\sigma_{B C_{1}}^{2}+\sigma_{B C_{2}}^{2}\right)
\end{aligned}
$$

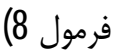

$$
\begin{aligned}
& \mathrm{D}=\left(\sigma_{B C_{1}}^{2}+\sigma_{B C_{2}}^{2}-\sigma_{F_{2}}^{2}-\sigma_{E}^{2}\right)
\end{aligned}
$$

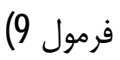

معتدل را در كلخانه و شرايط مزرعه بررسى كردند، در كلخانه

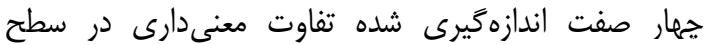

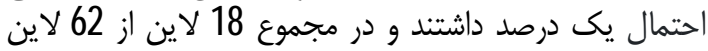

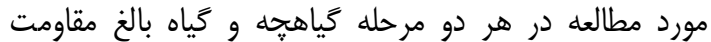

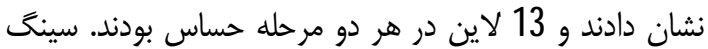

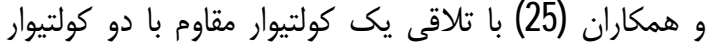

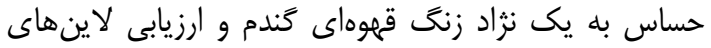

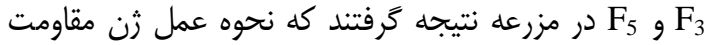

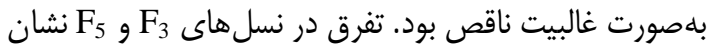

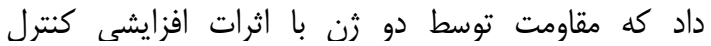

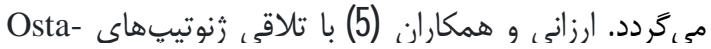

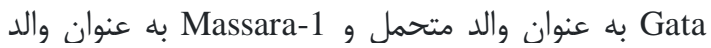

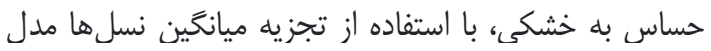

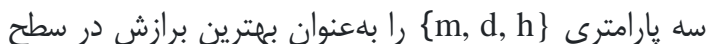

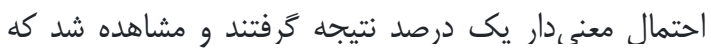

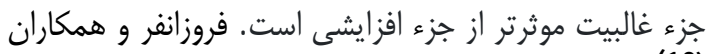

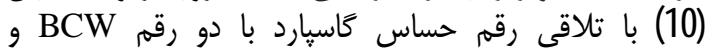

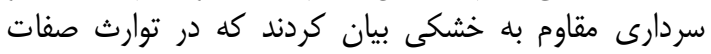

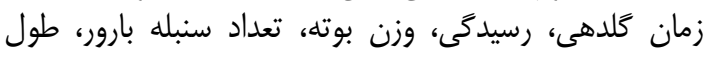

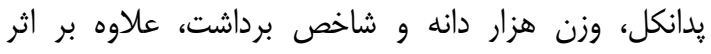

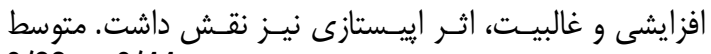

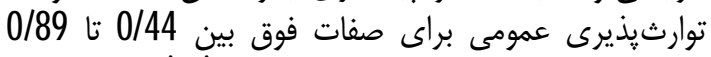

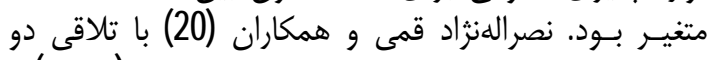

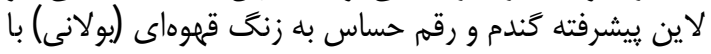

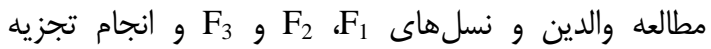

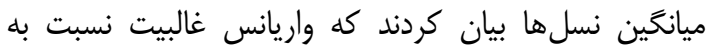

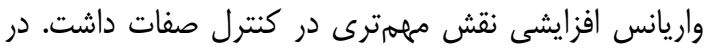

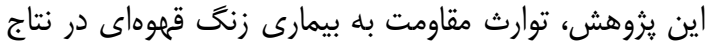

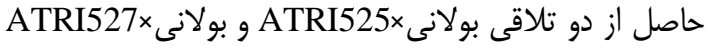

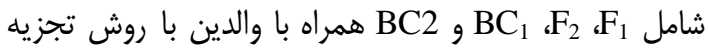
ميانگين نسل ها مورد بررسى قرار گرفت.

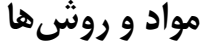

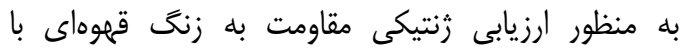

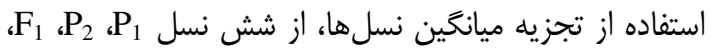

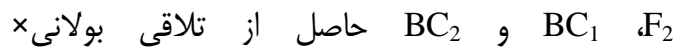

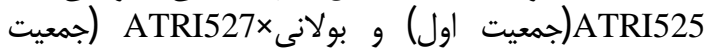
دوم) كه منشأ رقم بولانى (فاقد شجره) حساس بله به بيمارى

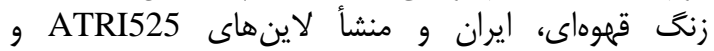

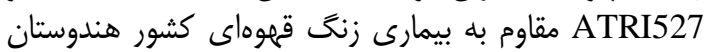

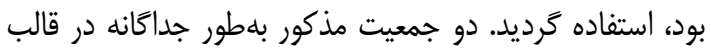
طرح بلوكهاى كامل تصادفى در ايستخاه تحقيقاتى كر كان دان در

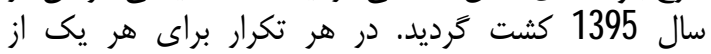

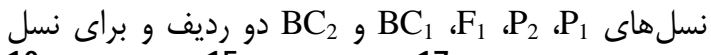

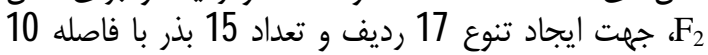

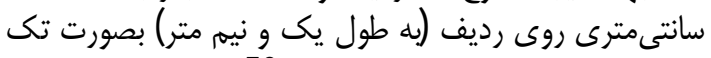

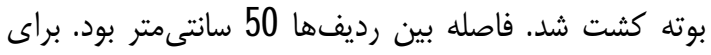

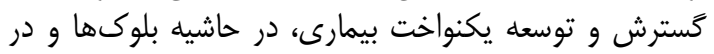
بين هر شش خط، يك خط رقم حساس بولانى بلهنوانوان 


$$
\begin{aligned}
& h_{B S}^{2}=\frac{\left[\widehat{\sigma}_{F 2}^{2}-\left(\sqrt[3]{\widehat{\sigma}_{p 1}^{2} \times \widehat{\sigma}_{P 2}^{2} \times \widehat{\sigma}_{F 1}^{2}}\right)\right]}{\widehat{\sigma}_{F 2}^{2}} \quad(14 \text { فرمول } \\
& h_{B S}^{2}=\frac{\left[\widehat{\sigma}_{F 2}^{2}-\left(\frac{\left(\hat{\sigma}_{P 1}^{2}+\widehat{\sigma}_{P 2}^{2}+\widehat{\sigma}_{F 1}^{2}\right)}{3}\right)\right]}{\widehat{\sigma}_{F 2}^{2}} \\
& h_{N S}^{2}=\frac{\left(2 \widehat{\sigma}_{F 2}^{2}-\widehat{\sigma}_{B C 1}^{2}+\widehat{\sigma}_{B C 2}^{2}\right)}{\widehat{\sigma}_{F 2}^{2}}
\end{aligned}
$$

تجزيه واريانس دادهها بر اساس آزمون مقياس مشترك با مرنا

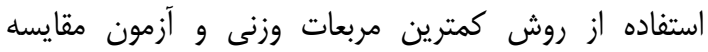

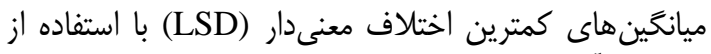
نرم افزار آمارى SAS إنجام كرين اختاف معنى

\section{نتايج و بحث}

نتايج حاصل از تجزيه واريانس دادهها بر اساس آزمون

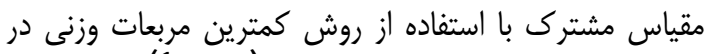

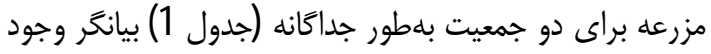

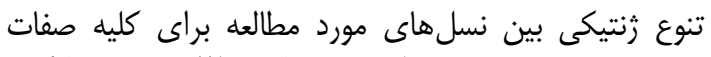

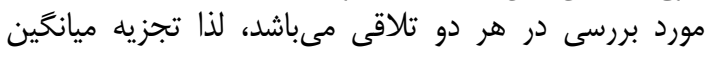
نسل ها امكان يذير است.
$\mathrm{F}=\left(\sigma_{B C_{2}}^{2}+\sigma_{B C_{1}}^{2}\right)$

فرمول10) - 2 (10)

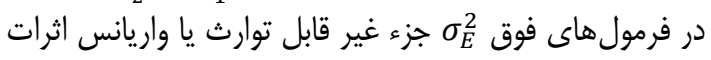

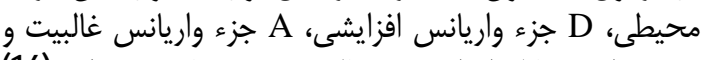

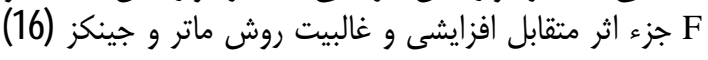

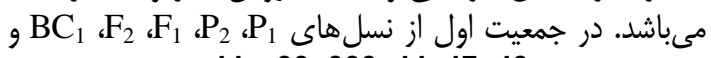

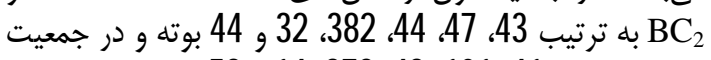

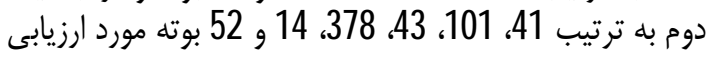

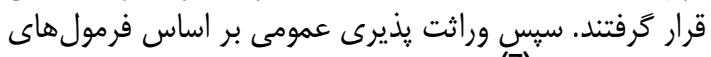

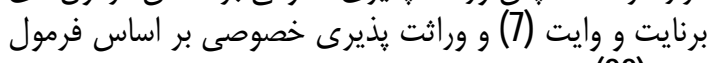
وارنر (28) محاسبه شد.

$h_{B S}^{2}=\frac{\left[\widehat{\sigma}_{F 2}^{2}-\left(\frac{\widehat{\sigma}_{P 1}^{2}+\widehat{\sigma}_{p 2}^{2}}{2}\right)\right]}{\widehat{\sigma}_{F 2}^{2}}$

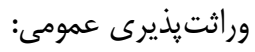

$$
\begin{aligned}
h_{B S}^{2} & =\frac{\left[\widehat{\sigma}_{F 2}^{2}-\left(\sqrt{\widehat{\sigma}_{P 1}^{2} \times \widehat{\sigma}_{P 2}^{2}}\right)\right]}{\widehat{\sigma}_{F 2}^{2}} \\
h_{B S}^{2} & =\frac{\left[\widehat{\sigma}_{F 2}^{2}-\left(\widehat{\sigma}_{F 2}^{2}\right)\right]}{\widehat{\sigma}_{F 2}^{2}}
\end{aligned}
$$

\begin{tabular}{|c|c|c|c|c|c|c|}
\hline \multicolumn{4}{|c|}{ ميانكين مربعات صفات } & \multirow{2}{*}{ درجه آزادى } & \multirow{2}{*}{ منابع تغييرات } & \multirow{2}{*}{$\dot{\lambda}_{\hat{y}}$} \\
\hline AUDPC & ACI & IT & PS & & & \\
\hline $29414=-166$ & $25 \mathrm{C} / 6$ & $7 / 66$ & $7 \mathbb{1} / 14$ & 2 & تكرار & \\
\hline 1460350E/6 & $2680 / \pi$ & 306/35 & 3319E/5/ & 5 & نسل & $\left.x^{2}\right)^{n}$ \\
\hline 3355$] / 2$ & $46 / 01$ & C/6I & $T_{4} / 91$ & IC & خطbا & $x^{3} \vec{z}$ \\
\hline 3E/7C & З/ع4 & $3 / / 3 C$ & 3/6 & - & ضريب تغييرات & \\
\hline 72921/74 & $116 / 85$ & $5 / 66$ & $316 / 06$ & 2 & تكرار & \\
\hline $14 / 23 / 01 / 45$ & $2664 / / 15$ & $318 / 96$ & $32586 / 0 E$ & 5 & نسل & 주 \\
\hline $12 / 54 / 32$ & $15 / E$ & $\mathrm{C} / 4 \mathrm{C}$ & 3]/OE & IC & خطا & $x^{3}$ \\
\hline$\chi / 15$ & E/E & $\equiv 4 / 32$ & $26 / 15$ & - & ضريب تغييرات & \\
\hline
\end{tabular}

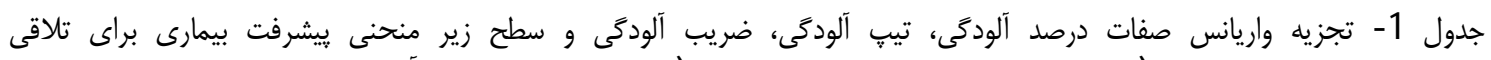

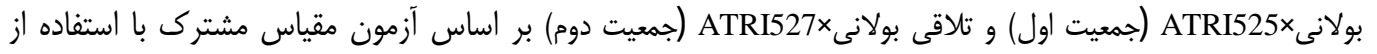

$$
\text { روش كمترين مربعات وزنى (رئى }
$$

Table 1. Analysis of variance of traits Percentage of contamination, infection type, contamination coefficient and area under the disease progression curve for the cross of BolanixATRI525 (first population) and cross of BolanixATRI527 (second population) based on joint scale test using weighted least square method

(ATRI527 نسبت به والد حساس (بولانى) داراى مقدار

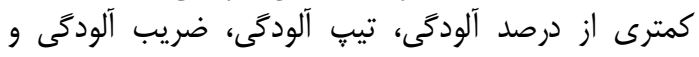

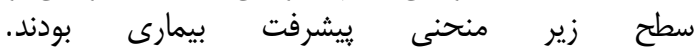

نتايج مقايسه ميانگَين شش نسل فوق (جدول 2) نشان

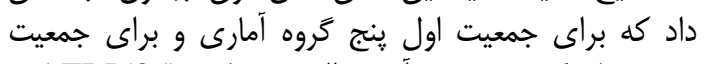
دوم جهار كروه بدست آمد. والدين مقاوم (ATRI525 واري 


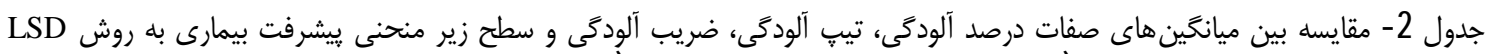

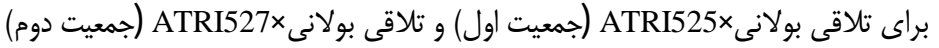

Table 2. Comparison between mean of traits, percentage of infection, infection type, contamination coefficient and area under the disease progression curve by LSD method for cross of BolanixATRI525 (first population) and cross of BolanixATRI527 (second population)

\begin{tabular}{|c|c|c|c|c|c|c|c|c|c|c|}
\hline \multirow{2}{*}{\multicolumn{4}{|c|}{ تلاقى بولانى ATRI527 }} & 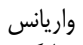 & \multicolumn{4}{|c|}{ تلاقى بولانى ATRI525 } & \multirow{3}{*}{ موانكين } & \multirow{3}{*}{ نسل } \\
\hline & & & & ميانكين & & & & & & \\
\hline AUDPC & ACI & IT & PS & نسل & AUDPC & ACI & IT & PS & & \\
\hline $1602 / 44^{a}$ & $6 / \alpha^{a}$ & $7 / 0 r^{a}$ & $7 / / O^{a}$ & C/35 & $160 / \sigma^{a}$ & $6=11^{a}$ & $7 / \mathbb{L}^{a}$ & $7 \varepsilon / 41^{a}$ & $C / 4 C$ & $P_{1}$ \\
\hline $145 / 3^{\mathrm{a}}$ & $1 / 5 e^{\mathrm{e}}$ & $\mathrm{C} / 22^{\mathrm{a}}$ & $7 / \mathbb{Q}^{\mathrm{u}}$ & $\mathrm{c} / 0$ & $112 / 9^{\mathrm{e}}$ & $1 / 2{ }^{\mathrm{e}}$ & $\mathrm{C}$ & $6 / 3^{2 e}$ & $C / O E$ & $\mathrm{P}_{2}$ \\
\hline $61 / 6^{\circ}$ & $1 \equiv / 8 /^{\circ}$ & $\exists / 0^{D}$ & $36 / \mathbb{L}^{\circ}$ & $C / \pi$ & $68=/ 9^{\circ}$ & $15 / 0^{c}$ & $\Xi^{\mathrm{D}}$ & $35 / 69^{\circ}$ & C/\& & $\mathrm{F}_{1}$ \\
\hline $296 / 1 c^{\mathrm{c}}$ & $4 / 9 q^{\mathrm{a}}$ & $1 / 2 c^{c}$ & $17 / 5^{c}$ & C/IC & $354 / 0 E^{c}$ & $\epsilon / \mathbb{T}^{\mathrm{a}}$ & $1 / 5 C^{\circ}$ & $1 \mathrm{C} / \mathrm{CC}^{\mathrm{C}}$ & C/3 & $\mathrm{F}_{2}$ \\
\hline $6 / \varepsilon / 5 T^{\circ}$ & $1 \mathcal{C} / 0 \bar{I}^{\circ}$ & $\Xi / 3^{50}$ & $35 / 6^{0}$ & $31 / 58$ & $71 C / 16^{\circ}$ & $2 / 5^{-0}$ & $\Xi / \mathbb{Z}^{0}$ & $36 / 9^{\circ}$ & $\varepsilon / 36$ & $\mathrm{BC}_{1}$ \\
\hline $26 / \varepsilon^{c}$ & $\Xi \mathrm{d}^{\mathrm{e}}$ & $\mathrm{C} / 9 \mathrm{~L}^{\circ}$ & $14 / \mathbb{Q}^{\mathrm{c}}$ & $\mathrm{C} / \mathrm{E}=$ & $232 / 1 \mathrm{C}^{\mathrm{a}}$ & $\Xi / 2 \mathrm{C}^{\mathrm{ue}}$ & $\mathrm{C} / 8 \mathrm{a}^{\mathrm{a}}$ & $12 / 84^{a}$ & $1 / 5 C$ & $\mathrm{BC}_{2}$ \\
\hline $4 c / 74$ & $1 / \pi$ & $C / Z \mathcal{E}$ & $2 / 45$ & - & $T \equiv / O E$ & $2 / \pi$ & C/32 & $\exists / 4 C$ & - & LSD \\
\hline
\end{tabular}

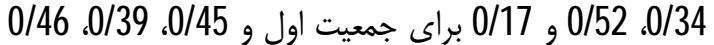

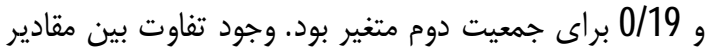
وراثتيذيرى عمومى و خصوصى در تلاقى بيانكر سهرم بيشتر

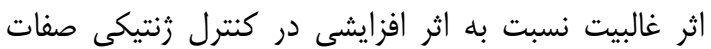

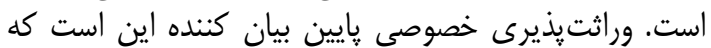

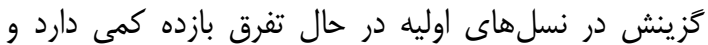

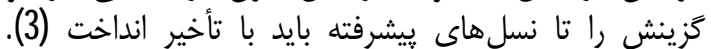

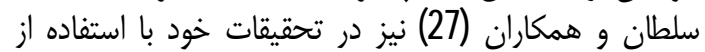

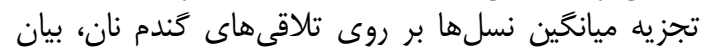

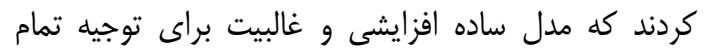

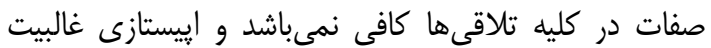
براى توجيه صفات تعداد دانه در سنبله، عملكرد دانه داند بر بوته

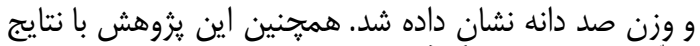

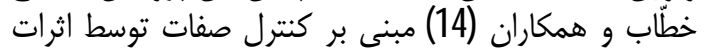

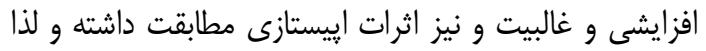
كزينش براى اين صفات در نسل هاى بعدى اصلاحى مؤثرتر خواهد بود.
بر اساس انحراف F⿸ از ميانكين والدين، درجه غالبيت

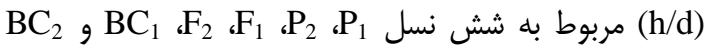

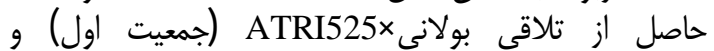

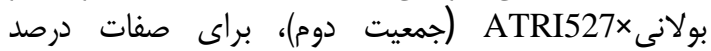

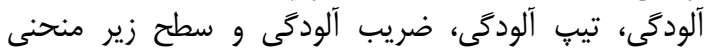

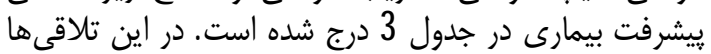

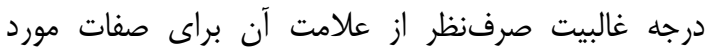

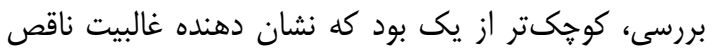

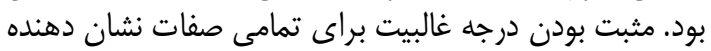

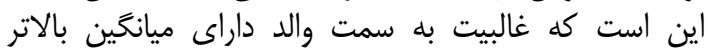

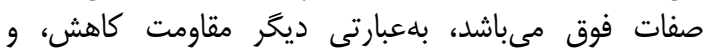

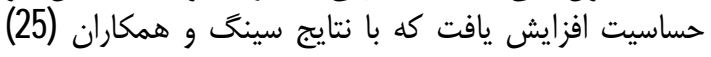

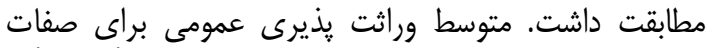

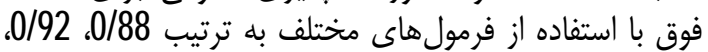

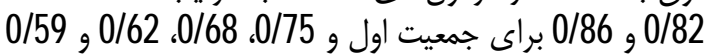

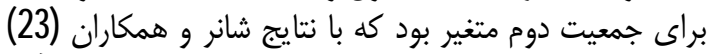
مطابقت داشت. وراثتيذيرى خصوصى نيز به ترتيب 0/42،

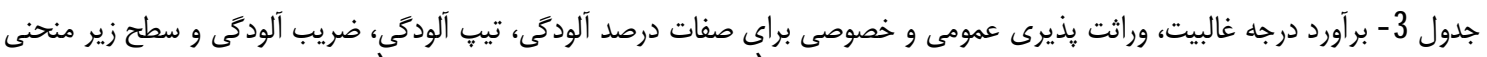

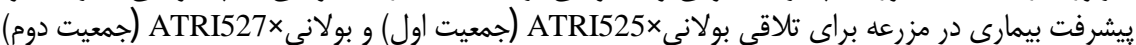

Table 3. Estimation of the degree of dominance, broad-sence heritability and narrow-sence for traits, percentage of infection, infection type, contamination coefficient and area under the disease progression curve for the cross of BolanixATRI525 (first population) and cross of BolanixATRI527 (second population)

\begin{tabular}{|c|c|c|c|c|c|c|c|c|c|}
\hline \multirow{2}{*}{ وراثتيذيرى خصوى } & \multirow[b]{2}{*}{ ميانكين } & \multicolumn{5}{|c|}{ وراثتيذيرى عمومى } & \multirow[b]{2}{*}{$\mathrm{h} / \mathrm{d}$} & \multirow[b]{2}{*}{ صفات } & \multirow{2}{*}{$\hat{x}_{\hat{n}}$} \\
\hline & & 5 & 4 & $\equiv$ & 2 & ] & & & \\
\hline $\mathrm{C} / 42$ & C/\&्E & C/8/ & $C / 96$ & C/6ॄ & C/91 & $C / 9$ & $\mathrm{C} / 5 \mathrm{C}$ & PS & \\
\hline C/3A & $\mathrm{C} / 92$ & $\mathrm{C} / \mathscr{C}$ & 1 & $C / \pi$ & 1 & C/9E & C/5/ & IT & $x^{2} \approx$ \\
\hline C/5z & C/82 & $\mathrm{C} / 6 \Subset$ & $\mathrm{C} / 96$ & C/6 & C/9E & $\mathrm{C} / \mathrm{BC}$ & $\mathrm{C} / 4 \mathrm{C}$ & $\mathrm{ACI}$ & \\
\hline C/17 & $C / \&$ & $C / T$ & C/94 & $C / \sigma$ & $(191$ & $\mathrm{C} / 9 \mathrm{I}$ & C/6 & AUDPC & \\
\hline$C / 45$ & $C / T$ & $\mathrm{C} / 6$ & $\mathrm{C} / \mathrm{T}$ & $C / 56$ & $C / \mathscr{C}$ & $C / 9$ & C/SE & PS & \\
\hline$C / 3 C$ & $C / \notin$ & $C / 54$ & $\mathrm{C} / 74$ & $C / 6=$ & $C / \pi$ & $C / T_{L}$ & C/61 & IT & \\
\hline$C / 4 C$ & $\mathrm{C} / \mathbf{Q}$ & $C / 6 \subset$ & $C / \pi$ & C/4C & C/8/ & C/3C & C/3/ & ACI & $x^{y} \cong$ \\
\hline C/ב & $C / 5 C$ & C/3A & $C / \pi$ & C/17 & C/8/ & C/84 & $C / \Phi$ & AUDPC & \\
\hline
\end{tabular}

آلودگىى، ضريب آلودگى و سطح زير منحنى ييشرفت بيمارى

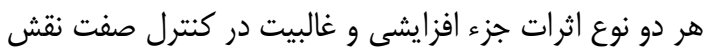

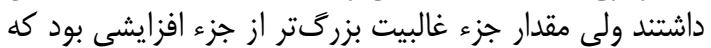

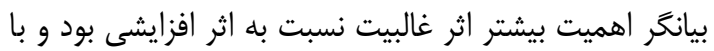

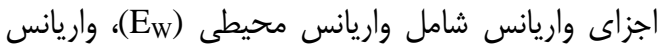

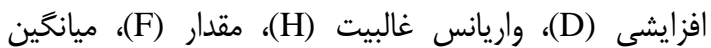
درجه غالبيت

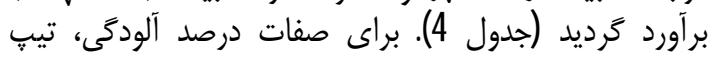


غالبيت در كنترل صفات مىباشد. قدر مطلق انحراف از

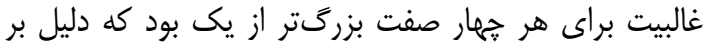

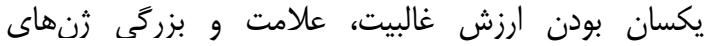
كنترل كننده صفات مذكور است و با نتايج ارزانى و همكاران

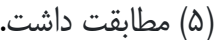

نتايج احمدى و همكاران (T) مطابقت داشت. در تلاقى

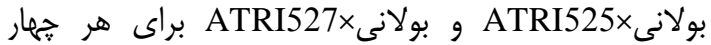

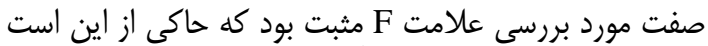

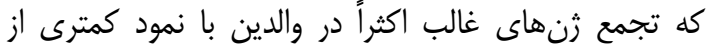

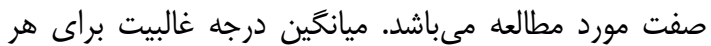

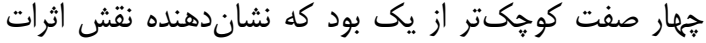

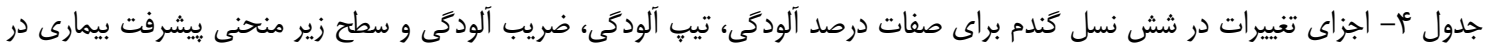

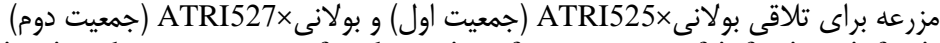

Table 4. Components of changes in six wheat genotypes for the traits of percentage of infection, infection type, contamination coefficient and area under the disease progression curve for cross of BolanixATRI525 (first population) and cross of Bolani×ATRI527 (second population)

\begin{tabular}{|c|c|c|c|c|c|c|c|}
\hline \multicolumn{6}{|c|}{ اجزاى تغييرات } & \multirow{2}{*}{ صفات } & \multirow{2}{*}{$\overbrace{\hat{x}}$} \\
\hline $\mathrm{F} / \sqrt{\mathrm{H} / \mathrm{D}}$ & $\sqrt{\mathrm{H} / \mathrm{D}}$ & $\mathrm{F}$ & EW & $\mathrm{H}$ & $\mathrm{D}$ & & \\
\hline קצr & $\cdot / V \cdot$ & THa/T. & $|q| \cdot r$ & $T F / \& D$ & $I T / F V$ & PS & \\
\hline$r / \Delta V$ & $\cdot / \Delta V$ & $r / \cdot r$ & .1 .9 & I/AT & $\mid /{ }_{1}$ & IT & $2 i$ \\
\hline FTN/FI & سو|. & req/a & $\mid r / \cdot r$ & سז/rس & $1 \pi / 4$ & ACI & \\
\hline$|r F| \cdot V / I V$ & $\cdot / \mathrm{V} \wedge$ & १९八.r/द & $V r V / \cdot 1$ & FVN $/ \cdot \Delta$ & $r q 4 / 4 \Delta$ & AUDPC & \\
\hline$|f q / r|$ & $\cdot / V q$ & $\| \pi / 4$ & $I T / F V$ & $|r / \Delta|$ & V/rq & PS & \\
\hline $1 / 19$ & $\cdot / \mathrm{VA}$ &.$/ 91$ & (1/ & سזאו & $\cdot / M$ & IT & $x^{2} \tilde{n}$ \\
\hline ५q/. &.$/ 8$. & TH/Ft & IT/RE & $|\varepsilon| \cdot V$ & $9 / \cdot \Delta$ & $\mathrm{ACI}$ & $\times 6$ \\
\hline DIE. T/KT & س & FrATq/AD & V\&q\&/א. & TET/VG & $|\Lambda| / ৭ \Delta$ & AUDPC & \\
\hline
\end{tabular}

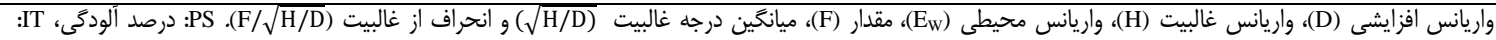

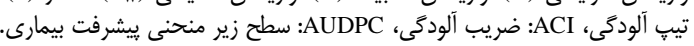

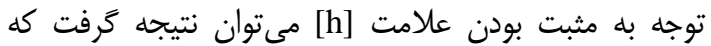

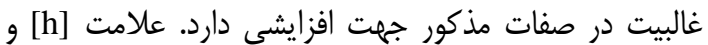

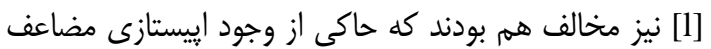

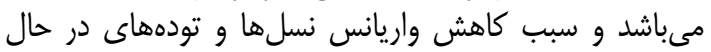

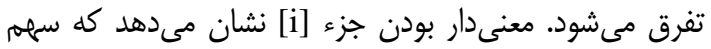

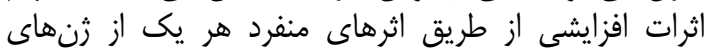

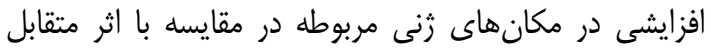

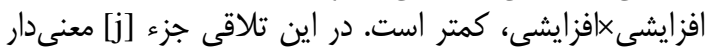

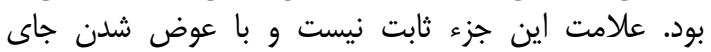

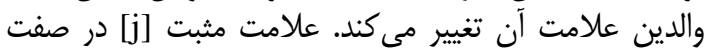

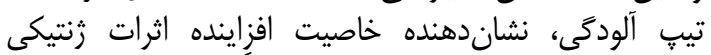

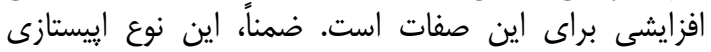

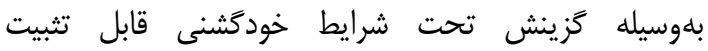

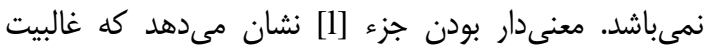

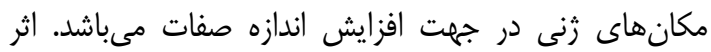

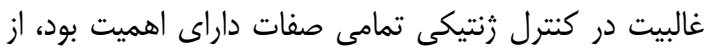

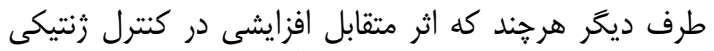

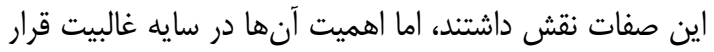

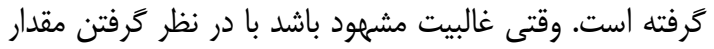

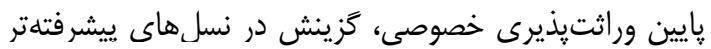

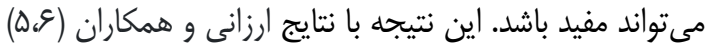

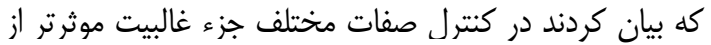

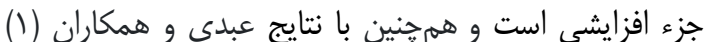

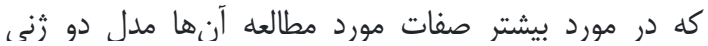

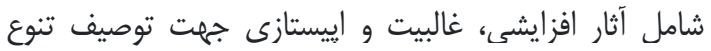
ميانكَين نسل ها مناسب بود، مطابقت داشت.
در اين تحقيق همه مدلهاى دو، سه، جهار، ينج و شش يُ براز

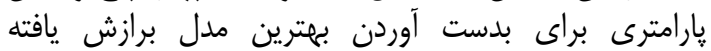

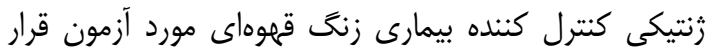

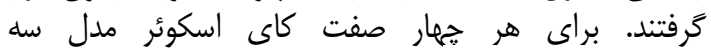
يارامترى

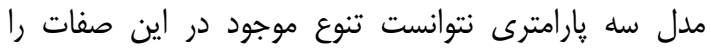

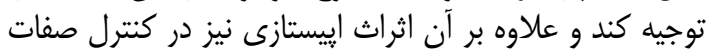

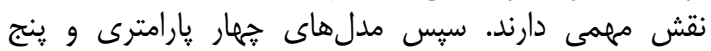

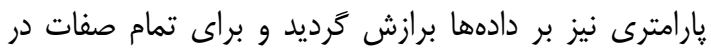

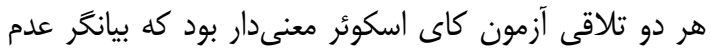

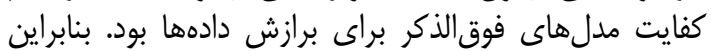

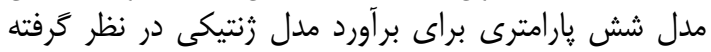

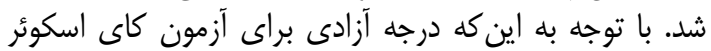

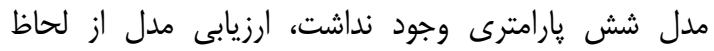

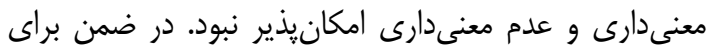

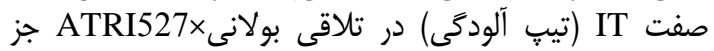

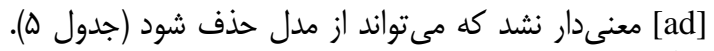

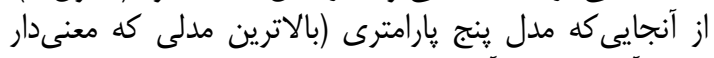

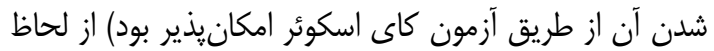

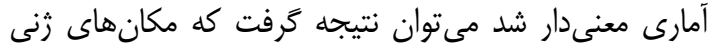

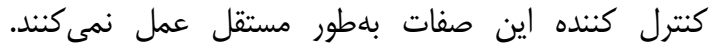

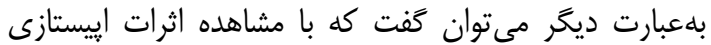

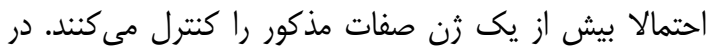

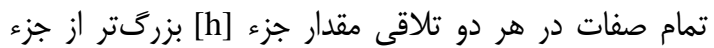

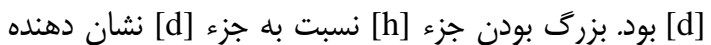

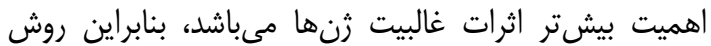
كزينش براى صفات در نسل هاى اوليه قابل استفاده نيست. باتئ 


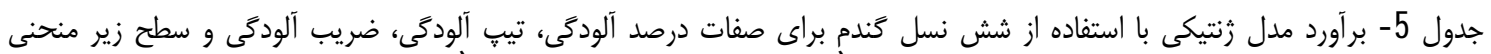

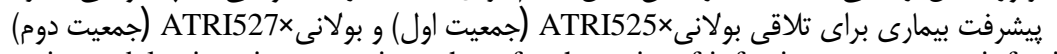

Table 5. Estimation of genetic model using six generation wheat for the traits of infection percentage, infection type, contamination coefficient and area under the disease progression curve for the cross BolanixATRI525 (first population) and cross BolanixATRI527 (second population)

\begin{tabular}{|c|c|c|c|c|c|c|c|c|}
\hline \multicolumn{7}{|c|}{ اجزاى رنتيكى } & \multirow{2}{*}{ صفات } & \multirow{2}{*}{ :ंके } \\
\hline $2 x$ & [ad] & [dd] & [aa] & [h] & [d] & {$[\mathrm{m}]$} & & \\
\hline $20=/ \bar{C}$ & 3/3/4 $8 / 2$ & $-11 / 1 / \pm 2 / 1 \varepsilon$ & $21 / 8 / \pm 4 / 35$ & $15 / 35+4 / 6$ & $24 / 8 c+1 / 95$ & $21 / 6 \pm(/ 6 c$ & PS & \\
\hline $9 / / \pi$ & $2 / 54 \pm \mathrm{C} / 8$ & $-1 / 0 C \pm C / 2$ & $2 / 31 \pm(/ 41$ & $1 / 8 \pm \pm(/ 4 /$ & $2 / 51+C / C^{\prime \prime}$ & $1 / 6 / \pm C / O c$ & IT & \\
\hline $20 / / 2$ & $2 E / 6 \pm 6 / 9=$ & - 1三/1Z+ 1/TE & $2 / / 3 / \pm \equiv / 46$ & $4 / 4 \pm \Xi / 6$ & $17 / 7 \pm \pm 1 / 58$ & $7 / 91+C / 52$ & ACI & \\
\hline 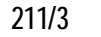 & $686 / 5 c+187 / 2$ & $-25 / 27+46 / 8$ & $507 / 4 E+9=/ 51$ & $33 / / 0= \pm 99 / 44$ & $49 E / 3 z \pm 42 / T_{2}$ & $38 / / 12 \pm 14 / 27$ & AUDPC & \\
\hline $2 \pi / 6$ & $26 / 8 \pm 7 / 1 C$ & $-1 \equiv / 14 \pm 1 / \pi$ & $3 \mathrm{C} / 04 \pm \pm / 54$ & $24 / 0 k \pm \equiv / 6 €$ & $2 / / 6 / \pm 1 / ஞ$ & $16 / 1 \varepsilon \pm(/ 41$ & PS & \\
\hline $107 / 6$ & $1 / 17 \pm(1 / 8]^{n s}$ & $-C / 9 C+C / X$ & $\Xi / 4 C \pm C / 4 C$ & $2 / \mathscr{C}_{C} \pm(/ 4 \mathrm{Z}$ & $2 / 5 C+C / C^{-\cdots}$ & $1 / 0 E+C / O$ & IT & \\
\hline $2016 / 2$ & $Z \approx / 0 E+\Delta / 94^{-\pi}$ & $-14 / 42+1 / 2$ & $24 / 4 \pm 2 / 4 \epsilon^{-\pi}$ & $6 / 14 \pm 2 / 5 C^{-1}$ & $16 / 3 c+1 / 1 T^{2}$ & $4 / 1 C+C / X^{m}$ & ACI & \\
\hline $31212^{* *}$ & $450 / 52+143 / 91^{* *}$ & $-314 / 21+35160^{* *}$ & $702 / 77 \pm 71 / 92^{* *}$ & $482 / 30+74 / 6{ }^{*}$ & $420182+34109^{* *}$ & $276 / 91+847^{* *}$ & AUDPC & \\
\hline
\end{tabular}

$\bar{\chi}_{\alpha=0.05, d f=3}^{2}=7.81$

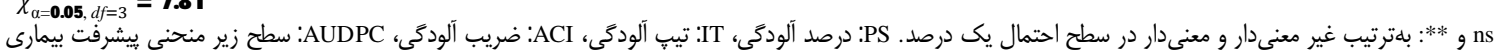

$$
\text { طول اجراى اين آزمايش تشكر و قدردانى مىشود. }
$$

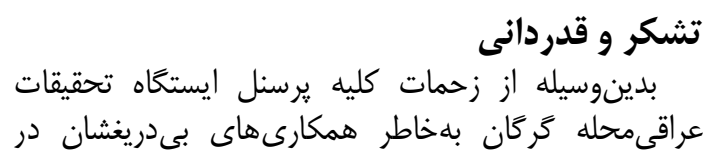

1. Abdi, H., M.H. Fotokian and S. Shabanpour. 2016. Studying the inheritance mode of grain yield and yield components in bread wheat genotypes using generations mean analysis. Cereal Research, 6(3): 283-292 (In Persian).

2. Ahmadi, J.S., F.A. Orang, A. Zali, B. Yazdi-Samadi, M.R. Ghanadha and A.R. Taleei. 2007. Study of yield and its components inheritance in wheat under drought and irrigated conditions. Journal of Science and Technology of Agriculture and Natural Resources, 11(1): 201-214 (In Persian).

3. Ahmadian, S., S.M.M. Mortazavian, M. Ebrahimi, F. Amini, M. Ghorbani Javid and B. Foghi. 2016. Genetic analysis of some morphological traits in wheat using generation mean analysis under normal and drought stress conditions. Journal of Crop Breeding, 8(2): 175-182 (In Persian).

4. Arzani, A. and M. Ashraf. 2017. Cultivated ancient wheats (Triticum spp): A potential source of health-beneficial food products. Comprehensive reviews in food science and food safety, 16(1): 477488.

5. Arzani, A., M. Golabadi and S.A.M. Mirmohammadimibadi. 2008. Genetic analysis of some morphological traits in durum wheat through mean generation analysis under stress and non-stress conditions. Seedling and Seeds, 24(1): 99-116 (In Persian).

6. Arzani, A., A. Ahoonmanesh and M. Torabi. 2005. A genetic study of adult plant resistance to Brown (Leaf) Rust in Bread Wheat. Iranian Journal of Agricultural, 36(2): 363-373 (In Persian).

7. Bernat, K. and J.N. Wright. 1985. A method for estimation heritability. Agron Journal, 44: 430-433.

8. Cavalli, L.L. 1952. An analysis of linkage in quantitative inheritance (Ed. E.C.R. Rieve and Waddington, C.H.) HMSO, Londan, pp: 135-144.

9. Dadrezaei, S.T., N. Tabatabai, I. Lakzadeh, A. Jafarnezhad, F. Afshari and Z. Hassanbayat. 2018. Evaluation of tolerance to leaf rust disease in some selected bread wheat genotypes. Entomology and Phytopathology, 86(1): 29-40 (In Persian).

10. Forouzanfar, M., M.R. Beyhmaeta, S.A. Peyghambari and H. Zainali. 2009. Inheritance of some traits associated with yield in bread wheat using generation mean analysis. Seedlings and seedlings, 125(3): 419-431 (In Persian).

11. Ghasemzade, E., F. Afshari, M. Khodarahmi and M. Bihamta. 2010. Study on the genetics of resistance to leaf rust in some advanced bread wheat lines at seedling stage. Journal of Agriculture and Breeding, 6(1): 51-59.

12. Hussain, F., M. Ashraf, A. Hameed Muhammad, N. Hussain and A. Sial Reza. 2011. Genetic studies in wheat for leaf resistance (Puccinia recondita). African Journal of Biotechnology, 10(16): 30513054 .

13. Hussain, M., S.F. Hassan and M.A.S. Kirmani. 1980. Virulence in Puccinia recondita f.sp .tritici in Pakistan during 1978 and 1979. Proceedings of the Fifth European and Mediterranean Cereal Rusts Conference, Bari, Italy: 179-184.

14. Khattab, S.A.M., R.M. Esmail and M.F. Abd EL-Rahman. 2010. Genetical analysis of some quantitative traits in bread wheat (Triticum aestivum L.) New York Science Journal, 3: 152-157. 
15. Mather, K. and J.L. Jinks. 1982. Biometrical genetics. The study of continuous variation. $3^{\text {rd }}$.edition. Chapman and Hall, Londan, New York, 365 pp.

16. Mather, k. and J.L. Jinks. 1949. Genetic and biometric foundation. First edition Chapman and Hall. London, $230 \mathrm{pp}$.

17. McIntosh, R.A., K.M. Devos, J. Dubcovsky, W.J. Rogers, C.F. Morris, R. Appels, D.J. Somers and O.A. Anderson. 2007. Catalogue of gene symbols for wheat: supplement. An Wheat News, 53: 159180.

18. McNeal, F.H., C.F. Konzak, E.P. Smith, W.S. Tate and T.S. Russel. 1971. A uniform system for recording and processing cereal research data. United States, Department of Agricultural Research Services, $42 \mathrm{pp}$.

19. Messmer, M.M., R. Seyfarth, M, Keller, G. Schachermayr, M. Winzeler, S. Zanetti, C. Fenillet and B. keller. 2000. Genetic analysis of durable leaf rust resistance in winter wheat. Theoretical and Applied Genetics, 100: 419-431.

20. Nasrollahnezhad Ghomi, A.A., M.R. Beyhmaeta, A. Hosseinzadeh, A.R. Talie, M. Torabi and M.R. Ghanadha. 2006. The inheritance of wheat brown rust resistance at adult stage. Iranian Journal of Agricultural Science, 1(1): 135-143 (In Persian).

21. Peterson, R.F., A.B. Campbell and A.E. Hannah. 1948. A diagrammatic scale for estimating rust intensity of leaves and stems of cereals. Canadian Journal of Research, 26: 496-500.

22. Roelfs, A.P., R.P. Singh and E.E. Saari. 1992. Rust diseases of wheat: Concepts and methods of disease management. CIMMYT, Mexico, D. F. 81 pp.

23. Shaner, G. and F.D. Hess. 1987. Equation for integration component of slow leaf rusting resistance in wheat. Phytopathology, 68: 1464-1469.

24. Sharma, P. and R.G. Saini. 2011. Genetics of durable resistance to leaf rust in bread wheat cultivars capelle desprez and pari 73. Journal of Phytology, 3(10): 10-15.

25. Singh, R.P., A. Mujeeb-Kazi and J. Huerta-Espino. 1998. Lr $r_{46}$ : A gene conferring slow-rusting resistance to leaf rust in wheat. Phytopathology, 88: 890-894.

26. Stubbs, R.W., J.M. Prescott, E.E. Saari and H.J. Dubin. 1986. Cereal disease methodology manual. CIMMYT, Mexico, D. F. 46 pp.

27. Sultan, M.S., A.H. Abd El-Latif, M.A. Abd El-Moneam and M.N.A. El-Hawary. 2011. Genetic parameters for some yield and yield components characters in four cross of bread wheat under two water regime treatments. Journal of Plant Production, 2: 351-366.

28. Warner, J.N. 1952. A method for estimation heritability. Agron Journal, 44: 427-430.

29. Wilcoxson, R.D., A.H. Atif and B. Skovmand. 1974. Slow rusting of wheat varieties in field correlated with stem rust severity on detached leaves in the greenhouse. Plant Disease, 58: 1058-1089.

30. Xu, X., G. Bai, B.F. Carver, G.E. Shaner and R.M. Hunger. 2005. Molecular characterization of slow leaf rusting resistance in wheat. Crop Science, 45: 758-765.

31.Zarandi, F., F. Afshari and S. Rezzaei. 2011. Virulence factors of Puccinia triticina the causal agent of wheat leaf rust in different parts of Iran. Seedling and Seeds, 1-27(2): 219-231 (In Persian). 


\title{
Inheritance of Resistance to Brown Rust Disease in Bread Wheat Via Means of Generation Analysis
}

\section{Hamid Ramroudi ${ }^{1}$, Ali Asghar Nasrollahnezhad Ghomi ${ }^{2}$, Khalil Zaynali Nezhad ${ }^{3}$ and Mohammad Ali Dehghan ${ }^{4}$}

\author{
1 and 3- M.Sc. Student and Assistant Professor, of Plant Breeding Student of Gorgan University of Agricultural \\ Sciences and Natural Resources \\ 2- Assistant Professor, Gorgan University of Agricultural Sciences and Natural Resources \\ (Corresponding author: nasrollahnejad@gau.ac.ir) \\ 4- Academic Member of Golestan Agricultural and Natural Resources Research and Education Center \\ Received: February 27, $2019 \quad$ Accepted: November 27, 2019
}

\begin{abstract}
Brown wheat rust caused by Puccinia recondita $\mathrm{f}$. sp tritici is one of the most important diseases of wheat in most parts of regions in the world. The progeny of $\mathrm{F}_{1}, \mathrm{~F}_{2}, \mathrm{BC}_{1}$ and $\mathrm{BC}_{2}$ derived from cross of Bolani×ATRI525 and Bolani×ATRI527 with parents, were cultured in a completely randomized block desigen for the study of wheat rust resistance. Trait such as infection, Infection type, contamination coefficient and surface under the disease progression curve were measured and evaluated. Genetic analysis by analysis of means and variance of generations showed that in addition to increasing effects, epistatic effects also play an important role in the control of traits. However, dominance variance had an important role in the control of traits in the incremental variance and there was a significant difference between the generations in terms of all traits in the one percent $(\mathrm{P}<0.01)$ level. The results of genotyping average analysis indicated that the dominance of genes was the most important genetic factor in control of the studied traits. Epistatic additivexadditive incremental addiction was more important than the epistatic dominancexdominance. The average of heritability for the traits was the percentage of infection, type of contamination, contamination coefficient and under curve of disease progression were $0.88,0.92,0.82$ and 0.86 for the first population and $0.75,0.68,0.62$ and 059 for the second population. Private heritability for the above mentioned traits was varied by 0.42 , $0.34,0.52$ and 0.17 for the first population, and $0.45,0.39,0.46$ and 0.19 for the second population. The mean genetic dominance $(\sqrt{ }(\mathrm{H} / \mathrm{D})$ was less than one for all four attributes, indicating the role of dominance effects in trait control.
\end{abstract}

Keywords: Epistatic, Dominance variance, Narrow-sence heritability, Broad-sence heritability 